\title{
Der Löß am unteren Mittel- und Niederrhein
}

\author{
Von Horst Remy, Bonn \\ Mit 7 Abbildungen im Text
}

\begin{abstract}
$\mathrm{Z}$ us a mmenf assung. Auf Grund der Beziehungen zwischen Löß und Unterer Mittelterrasse des Rheines und seiner Nebenflüsse läßt sich das Alter der Lößablagerungen im Mittelund Niederrheingebiet festlegen. In der Hauptsache handelt es sich dabei um Würmlöß, der von mehreren Bodenhorizonten gegliedert wird. Dabei waren im frühen Würm die Klimaänderungen noch beträchtlich (Parabraunerden), während der spätere klimatische Wechsel meist nur durch Naß̈böden gekennzeichnet ist. Lokal kam es in diesem späteren Abschnitt zur Bildung einer kalkhaltigen Braunerde, an die das Spätaurignac von Metternich geknüpft ist.

A bstract. In the Middle and Lower Rhine Valley the age of loess deposits is recognized by their relation to the Lower Middle Terrace of the Mosel and Rhine Rivers. Nearly all loess is regarded to be Würm-loess, which is separated by several soil zones. In the lower part of the Würm-period climatic changes are clearly indicated by thickly and we!l developed soil zones ("Parabraunerde"). Later on soil zones become generally thin and less distinct ("Naßböden"). In this later period, at some places, one soil zone is typical ("kalkhaltige Braunerde"). In the profile of Metternich this horizon included artifacts (Gravettian).
\end{abstract}

\section{Einleitung}

Im Niederrheingebiet überdeckt der Löß weite Flächen in zum Teil bedeutender Mächtigkeit, wie zahlreiche Aufschlïsse in Ziegeleigruben und Braunkohlentagebauen zeigen. Im Mittelrheinabschnitt hält sich der Löß in größter Mächtigkeit im wesentlichen an die Talhänge und ist auf den Hochflächen meist nur geringmächtig oder gar nicht vorhanden. Die vorliegende Arbeit soll einen ersten allgemeinen Uberblick über die Gliederung der Lößablagerüngen in diesem Raume geben und einen Ausgangspunkt für regionale Vergleiche schaffen.

Aus dem Mittel- und Niederrheingebiet liegen einige neuaufgenommene Lößprofile vor (E. Mücxenhausen 1954, 1959; E. A. Rosauer 1957; J. Frechen \& E. A. Rosauer 1959; H. REMY \& W. PAAS 1959; H. ReMY 1959, 1960). Weitere Profile werden hier dargestellt und kurz erläutert. Viele Profile, besonders die, die nur den jüngsten Löß aufschließen, sind fortgelassen worden. Es kam für einen allgemeinen Uberblick darauf an, nur die typischen und gut entwickelten herauszugreifen (Abb. 1). Die Lößprofile werden mit Hilfe von Bodenbildungen in mehrere Abschnitte (A bis $\mathrm{E}$ ) gegliedert. Die Einteilung in Alt-, Mittel- und Jungwürm wurde aus einer neuen Arbeit von Herrn Prof. P. WoLDSTEDT (in diesem Bande 1960) übernommen. Dabei entsprechen dem Altwürm die Abschnitte $A$ und $B$, dem Mittelwürm $C$ und $D$ und dem Jungwürm der Abschnitt $E$, wie sich das nach der Lagerung und nach den Kulturhinterlassenschaften des paläolithischen Menschen wahrscheinlich machen läßt.

Herr Prof. Dr. P. Woldstedt, Bonn, hat spezielle Untersuchungen immer wieder angeregt und gefördert und auch zu diesem kleinen Überblick den Anstoß gegeben. Herr Prof. Dr. Dr. E. MüCKENHAUSEN, Bonn, gab bei zahlreichen Geländebegehungen dem Verf. wertvolle Hinweise. Im Gebiet der Braunkohlentagebaue verdanke ich Herrn Dr. Heide, Krefeld, und dem Amt für Bodenforschung in Krefeld die Möglichkeit, Einblick in die Lößablagerungen zu gewinnen. Die Deutsche Forschungsgemeinschaft förderte durch finanzielle Unterstützung die Geländeaufnahmen. Allen Genannten möchte ich meinen aufrichtigen Dank aussprechen.

\section{Das Lößprofil von Koblenz-Metternich}

Dieses Profil ist erst kürzlich ausführlicher behandelt worden (H. REMY \& W. PAAS 1959). Einige Beobachtungen seien aber noch nachgetragen. Im Liegenden der Parabraunerden konnten vom Verf. die Terrassenschotter ergraben werden, so daß das Profil bis in den Schotterkörper der uMT zu verfolgen ist (Abb. 2). Am Ostabhang des Kimmel- 


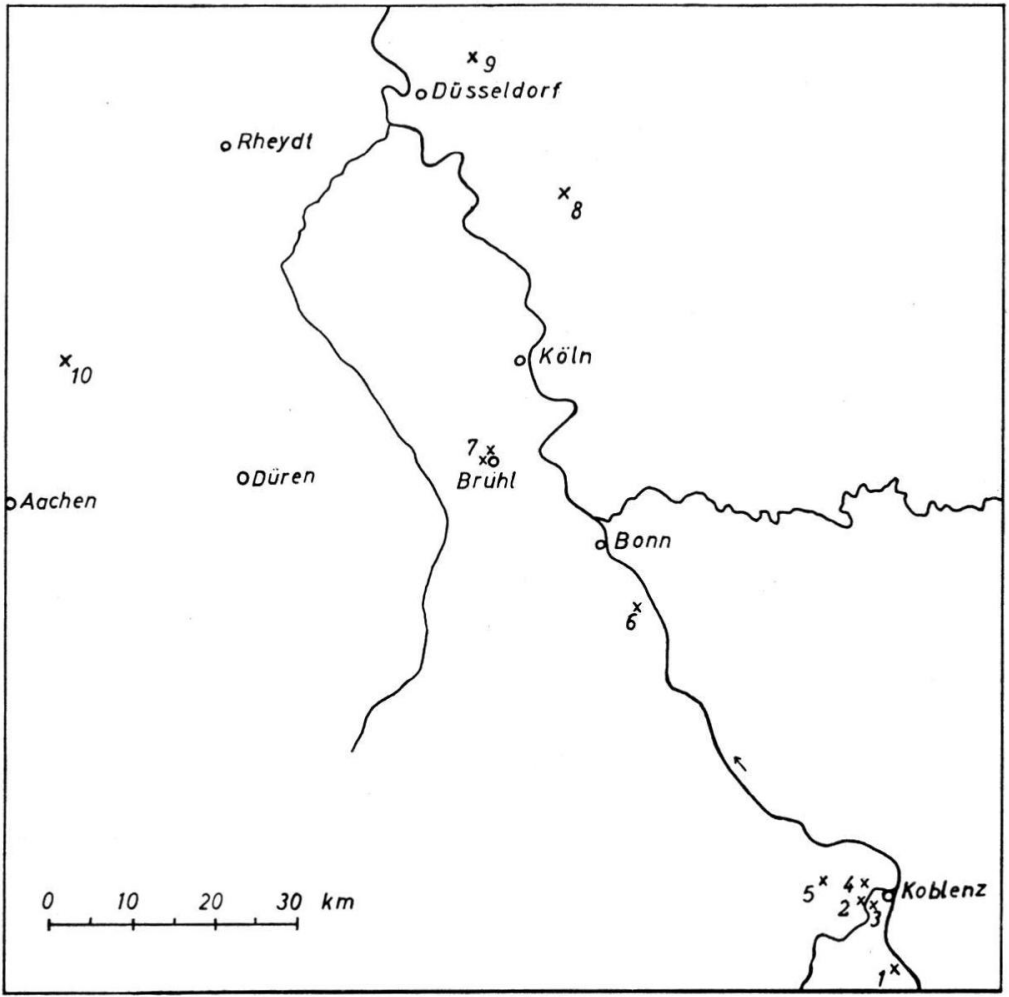

Abb. 1. Ubersichtsskizze zu den angeführten Lößprofilen: 1 Rhens, 2 Kobl.-Metternich, 3 Kobl.Moselweiß, 4 Kobl.-Metternich/Zgl. Friedhofen, 5 Kärlich, 6 Deusenberg b. Mehlem, 7 Brühl, Zgl. Garzen I u. II, 8 Leichlingen, 9 Düsseldorf-Grafenberg, $10 \mathrm{Zgl}$. Rosen, Beggendorf.

berges südlich Metternich liegen über Schottern der Unteren Mittelterrasse der Mosel (Schotter-Oberkante bei $85 \mathrm{~m}$ über NN) ca. $35 \mathrm{~m}$ Löß. Dieser ist in der ehemaligen Grube der Gebrüder Weglau zum größten Tejl auch heute noch aufgeschlossen und durch mehrere Bodenbildungen und Solifluktions-Einlagerungen gegliedert (Abb. 2):

Bis $0,95 \mathrm{~m}$ Braunerde des Spätglazials und Holozäns

A-Horizont: humoser, schwach lehmiger Grobsand (Laacher Bims-Tuff)

(B) 1 -Horizont: hellbrauner Grobsand (Bims-Tuff).

(B)2-Horizont: feinsandiger Lehm (Farbwert nach Munsell 1954: 7,5 YR

$\mathrm{E}$ 5/6), Bröckel- bis Polyeder-Gefüge

- 4,00 m Löß (10 YR 6/4), an der Basis Lößschnecken

- 4,60 m schwach verlehmter sandiger Löß, im unteren Teil wenige kleine Lößkindel (umgelagertes Bodenmaterial), an der Basis eine $1-2 \mathrm{~cm}$ starke Tuff-Lage.

- 6,45 m Löß (10 YR 6/4), darin Lage von schwach sandigem Löß mit Hangschutt.

- 7,55 m kalkhaltiger, feinsandiger Lehm (10 YR 5/6), an der Basis Lößkindel-Horizont: kalkhaltige Braunerde.

- 12,60 m Löß (10 YR 6/4)

- 13,40 m sandiger, kieselsteinreicher Löß mit Lößschnecken (10 YR 6/4): Fließerde.

- 14,00 m kalkhaltiger, feinsandiger Lehm (?umgelagerter Lößlehm) Lößschnecken.

C $\quad-17,80 \mathrm{~m} \mathrm{Löß} \mathrm{(10} \mathrm{YR} \mathrm{6/4)}$

- 23,35 m humoser, feinsandiger Lehm (10 YR 4/3) mit Einlagerungen von Hangschutt und Lößlehmmaterial: Fließerde. 
Parabraunerden, Braunerden und kalkhaltige Braunerden.

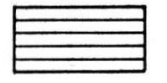

Naßböden

\section{$\div \div-$ Umgelagertes Material (Fließerden u. a.)}

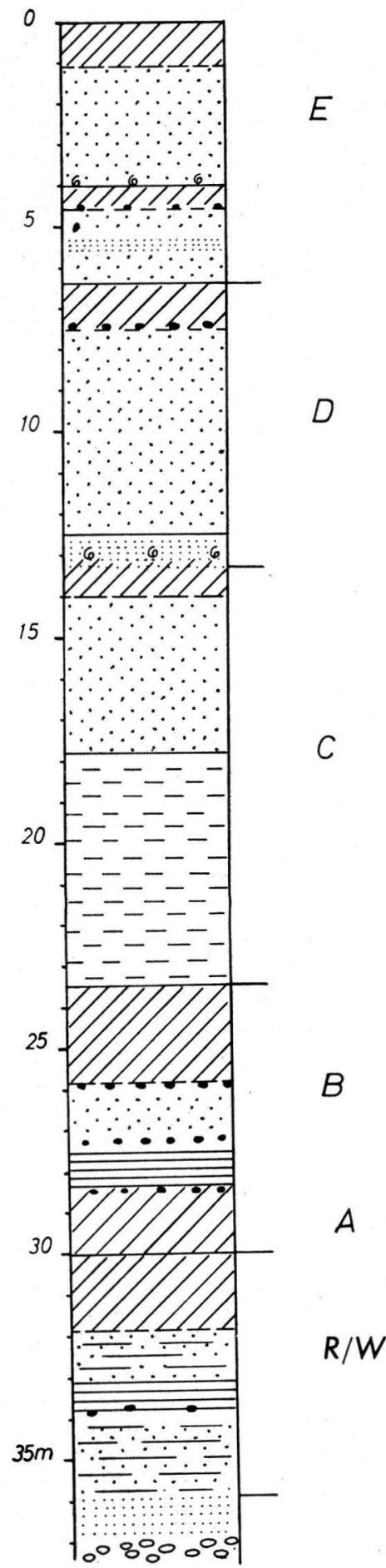

Abb. 2a. Erläuterung der Signaturen

Ca-Horizonte.

Sandeinlagerungen

Terrassenschotter
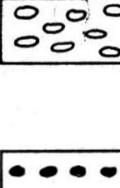

08000

Abb. 2. Lößprofil von Kobl.-Metternich, ehem. Zgl.-Grube Gebr. Weglau. 
- 25,95 m Parabraunerde mit starker Durchschlämmung:

$0,2 \mathrm{~m}$ A-Horizont: humoser, feinsandiger Lehm (10 YR 4/3)

B

1,45 m B-Horizont: feinsandiger Lehm mit wenigen Kieselsteinen (oben:

7,5 YR 4/4, unten: 7,5 YR 5/6), Tonhäutchen auf den Gefügeflächen, oben: Prismen-Gefüge; unten: Bröckel- bis Polyeder-Gefüge.

$0,95 \mathrm{~m}$ (B)-Horizont: streifiger, feinsandiger Lehm (10 YR 5/6), an der Basis Lößkindelhorizont.

- 27,60 m Löß mit wenigen Kieselsteinen (10 YR 6/4), an der Basis Lößkindel.

- 28,20 m grauer Lößlehm mit zahlreichen kleinen Eisenflecken, teilweise streifig, an der Basis Lößkindel: Naßboden.

- 29,95 m kalkhaltiger, feinsandiger Lehm (umgelagerter Lößlehm) (10 YR 5/4 und 10 YR $4 / 4)$.

- 31,85 m Parabraunerde mit mittlerer Durchschlämmung:

$0,60 \mathrm{~m}$ A-Horizont und A, kolluvial: Devon-Schutt mit humosem, feinsandigem Lehm (10 YR 4/3).

$\mathrm{R} / \mathrm{W}$

$0,30 \mathrm{~m}$ As-Horizont: Devon-Material mit feinsandigem Lehm (10 YR 5/4)

$1,00 \mathrm{~m}^{+}$B-Horizont: feinsandiger Lehm mit Devon-Material (7,5 YR 4-5/4), Tonhäutchen auf den Gefügeflächen, schwach fahlfleckig.

Wenige Meter von diesem Profil wurden in einer Bodensenke (alter Aushub) unter Fließlöß die Terrassenschotter der uMT angetroffen:

- 33,10 m schwach kalkhaltiger feinsandiger, streifiger Löß (10 YR 5/6)

- 33,70 m grauer, schwach kalkhaltiger Lößlehm, schwach eisenfleckig: Naßboden.

- 35,80 m geschichteter Löß (10 YR 5/8).

- 37,20 m kalkhaltiger Sand mit Schottermaterial.

In einer verlassenen Kiesgrube wenig unterhalb der Lößwand sind die Schotter der Unteren Mittelterrasse der Mosel aufgeschlossen. Diese tragen eine kräftige Verwitterung, die bodentypologisch dem Braunlehm nahesteht und etwas anderes darstellt als Parabraunerden im Löß (freundl. Mitt. von W. PAAs). Diese Verwitterung ist im Profil die intensivste Bodenbildung in der Zeit nach dem Saale-Maximum und würde am besten in das Letzte Interglazial (Eem) zu stellen sein; denn wir kennen nach dem Vorstoß des Saaleeises mit Sicherheit als echte Warmzeit vom Ausmaß eines Interglazials nur das Eem-Interglazial. Das würde bedeuten, daß alle Ablagerungen und Bodenbildungen über der Verwitterungsdecke der Unteren Mittelterrasse würmeiszeitliches Alter haben. So wahrscheinlich diese Deutung aber auf Grund des bodenkundlichen Befundes sein mag, so stehen dem andere Beobachtungen entgegen. Es wird daher in Metternich als würmzeitlich vorerst nur der Löß über der älteren Parabraunerde (29,95 bis $31,85 \mathrm{~m}$ ) aufgefaßt. Die älteren Bildungen gehören danach ins Jungriß.

Der Würm-Lößkomplex wird durch mehrere verschieden mächtige Bodenbildungen gegliedert. Im Bereich (A) liegt umgelagertes Bodenmaterial. Dieses könnte einer Bodenbildung entstammen, die in anderen Profilen in wohl gleicher stratigraphischer Position als kalkhaltige Braunerde i. S. von E. Schönhals vorliegt (Koblenz-Moseiweiß, Kärlich). Die Parabraunerde $(23,35$ bis $25,95 \mathrm{~m})$ darüber bezeichnet eine erneute nicht unerhebliche Erwärmung. Dieser Zeitabschnitt (A und B) wird als Altwürm aufgefaßt, da von der ehemaligen Ziegeleigrube Friedhofen in Metternich aus einem mit dem Abschnitt B zu parallelisierenden Horizont ein Artefakt der Moustier-Kultur stammt

Dem Komplex des Altwürms steht im oberen Teil der Profile ein Lößkomplex gegenüber, der von weniger mächtigen Böden gegliedert wird. Dieser Komplex umfaßt das Mittel- $(C+D)$ und Jungwürm (E).

Den basalen Teil des Mittelwürms bildet in Metternich eine mächtige Fließerde, die im wesentlichen aus der jüngeren Parabraunerde (B) hervorgegangen ist. Die darüberliegende jüngere Fließerde leitet einen besonderen Abschnitt (D) ein. Das Mittelwürm 
endet in Metternich mit einer deutlichen Bodenbildung (D), die im Sinne von E. SchöNHALS (1951) als kalkhaltige Braunerde bezeichnet wird. Aus diesem Horizont hat A. GüNTHER (1907) Werkzeuge des Spätaurignacs beschrieben. Die Werkzeuge lagen an der Oberfläche bzw. in einer in die Schicht eingetieften Mulde. Dieses geht auch aus der Aufnahme von H. Hofer (1937) hervor, der in späteren Jahren weitere Werkzeuge auffinden konnte.

Nach der Entstehung der kalkhaltigen Braunerde (D) muß es im Jungwürm (E) noch einmal zu einer schwachen Bodenbildung (4,0-4,6 m) gekommen sein. An der Basis dieser liegt ein Tuffbändchen, das einem Ausbruch bei Kärlich entstammen dürfte. Dieses findet sich auch in weiter südlich gelegenen Lößaufschlüssen über einer schwachen Verlehmungszone, die mit der kalkhaltigen Braunerde von Metternich $(6,45-7,55 \mathrm{~m})$ identisch sein könnte.

Gegen Ende der Lößbildungszeit wurde Laacher Bims-Tuff abgelagert, der auch in vielen anderen rheinischen Lößprofilen in dieser Position auftritt. Die spätglaziale und holozäne Verwitterung führte in Metternich zur Bildung einer ca. $1 \mathrm{~m}$ mächtigen Braunerde.

\section{Ziegeleigruben bei Brühl}

Am Osthang der Ville liegen am Ortsrande von Brühl die noch im Abbau stehenden Ziegeleigruben Garzen I und II (Abb. 3 u. 4). Im Liegenden werden Schotter der Unteren Mittelterrasse des Rheines angegeben. Die Profile bei Brühl fallen durch fluviatile Sandeinlagerungen im Mittel-Würmkomplex auf. Garzen I:

Bis $1,40 \mathrm{~m}$ Parabraunerde des Spätglazials und Holozäns $0,2 \mathrm{~m}$ A-Horizont: humoser, krümeliger Lößlehm

\section{$0,2 \mathrm{~m} \mathrm{~A}$-Horizont: feinsandiger Lößlehm (10 YR 5/4)}

E $\quad 0,7 \mathrm{~m}$ B-Horizont: feinsandiger Lößlehm (10 YR 5/6), Tonhäutchen auf den Gefügeflächen.

$0,3 \mathrm{~m}$ (B)-Horizont: feinsandiger Lößlehm (10 YR 5/4)

- $2,30 \mathrm{~m}$ Löß (10 YR 6/4)

- $2,95 \mathrm{~m}$ hellgrauer Löß mit kleinen Eisenflecken; darin Flugsandeinlagerungen.

- 3,50 m Löß (10 YR 6/4), leicht fleckig

- 4,10 m kalkhaltiger, feinsandiger Lehm, zum Teil schwach humos, kleine Eisenflecken

D (10 YR 5/4), an der Basis Lößkindel: Naßbaden

- 4,50 m Löß (10 YR 6/6)

- $4,95 \mathrm{~m}$ hellgrauer kalkhaltiger Löß mit kleinen Eisenflecken: Naßboden

- 6,00 m Löß (10 YR 6/4)

Hangwärts liegt eine mächtige Sand- bzw. Kieselsteinlage, die etwa in der Mitte des Aufschlusses auskeilt.

C $\quad-7,00 \mathrm{~m}$ kalkhaltiger, feinsandiger, schwach humoser Lößlehm (10 YR 5/6), im unteren Teil sandig und streifig, an der Basis Lößkindel: kalkhaltige Braunerde.

- $8,20 \mathrm{~m}$ sandiger, geschichteter Löß

- 9,00 m brauner fluviatiler Sand (oben: 7,5 YR 5/8; unten: 10 YR 5/6)

$-9,70 \mathrm{~m}+$ Parabraunerde, schwach pseudovergleyt

B B-Horizont: rötlich-brauner Lößlehm (10 YR 5/6), Tonhäutchen auf den Gefügeflächen, an der Oberfläche kleine Eisenmangankonkretionen.

Ganz ähnlich sind die Verhältnisse in Garzen II (Abb. 4):

Bis $1,00 \mathrm{~m}$ Bodenbildung des Spätglazials und Holozäns

- $1,40 \mathrm{~m}$ Löß (10 YR 6/4)

$\mathrm{E} \quad-1,70 \mathrm{~m}$ kalkhaltiger, sandiger Löß mit Hangschutt und Lößschnecken (10 YR 6/6) (schwach verlehmtes Lößmaterial): Fließerde

- $2,15 \mathrm{~m}$ Löß (10 YR 6/4)

- 2,80 m gelblich-brauner Sand (10 YR 5/6) 
- $3,25 \mathrm{~m}$ kalkhaltiger, feinsandiger Lehm (10 YR 5/6) mit zahlreichen Eisenflecken, an D der Basis Lößkindel: kalkhaltige Braunerde.

- $5,15 \mathrm{~m}$ Löß (10 YR 6/4)

- 5,55 m kalkhaltiger, feinsandiger Löß mit Eisenflecken, streifig, an der Basis Lößkindel.

- 6,40 m Löß (10 YR 6/4)

- 9,05 m geschichteter Löß mit Sandbändern (10 YR 6/4);

C bei 7,2 m: 0,1 m schwach grauer kalkhaltiger Löß mit wenigen Eisenflecken. bei $8,10 \mathrm{~m}-8,40 \mathrm{~m}$ : hellgrauer Löß mit zahlreichen Eisenflecken.

- 10,20 m brauner fluviatiler Sand (oben: 7,5 YR 5/8; unten: 10 YR 5/6).

\section{$-11,30 \mathrm{~m}+$ Parabraunerde}

$0,35 \mathrm{~m}$ : A-Horizont: humoser, feinsandiger Lehm (10 YR 4/3)

B

$0,2 \mathrm{~m} \mathrm{~A}$-Horizont: feinsandiger Lehm (10 YR 5/4)

$0,55 \mathrm{~m}^{+}$B-Horizont: feinsandiger Lehm mit Kieselsteinen (10 YR 5/6).

Die Profile bei Brühl fallen durch fluviatile Sandeinlagerungen im Mittel-Würmkomplex auf. Durch diese wie auch mehrere Naßbodenhorizonte wird der Abschnitt C in diesem Gebiet stärker als etwa in Metternich gegliedert. Hervorzuheben ist in Garzen I

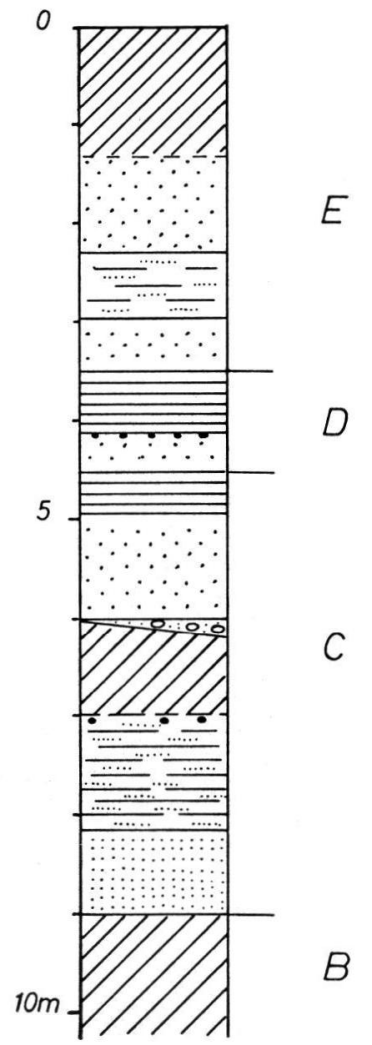

Abb. 3. Lößprofil von Brühl, Zgl. Garzen I

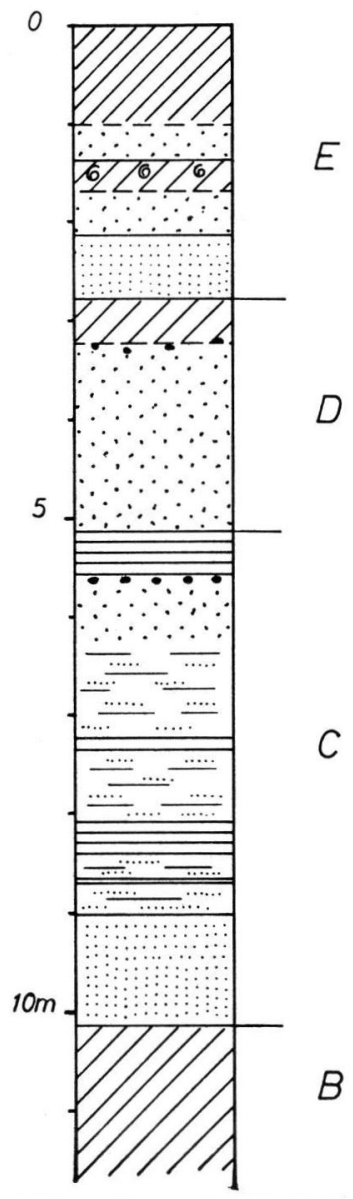

Abb. 4. Lößprofil von Brühl, Zgl. Garzen II. 
etwa in der Mitte von Abschnitt C die Bodenbildung, die im Sinne von E. Schönhals als kalkhaltige Braunerde bezeichnet wird. Die Lößkindel an der Basis weisen darauf hin, $\mathrm{da} ß$ es sich wahrscheinlich um eine autochthone Bildung handelt. In anderen Profilen ist dieser Horizont als Naßboden oder Fließerde entwickelt. Man könnte eventuell in Metternich den Rest einer ähnlichen Bodenbildung in dem offenbar umgelagerten Bodenmaterial im oberen Teil von C (13,40-14,00 m) sehen. Im Rheingau scheint es Profile zu geben, die eine kalkhaltige Braunerde in ähnlicher Position aufweisen (z. B. das Profil von Eltville).

In den beiden Profilen von Brühl haben wir im Jungwürm (E) wieder die Andeutung einer Klimaschwankung: In Garzen I liegt eine schwache Naßbodenbildung (2,30 bis $2,95 \mathrm{~m})$, in Garzen II eine Fließerde $(1,4-1,7 \mathrm{~m})$ in diesem Abschnitt vor.
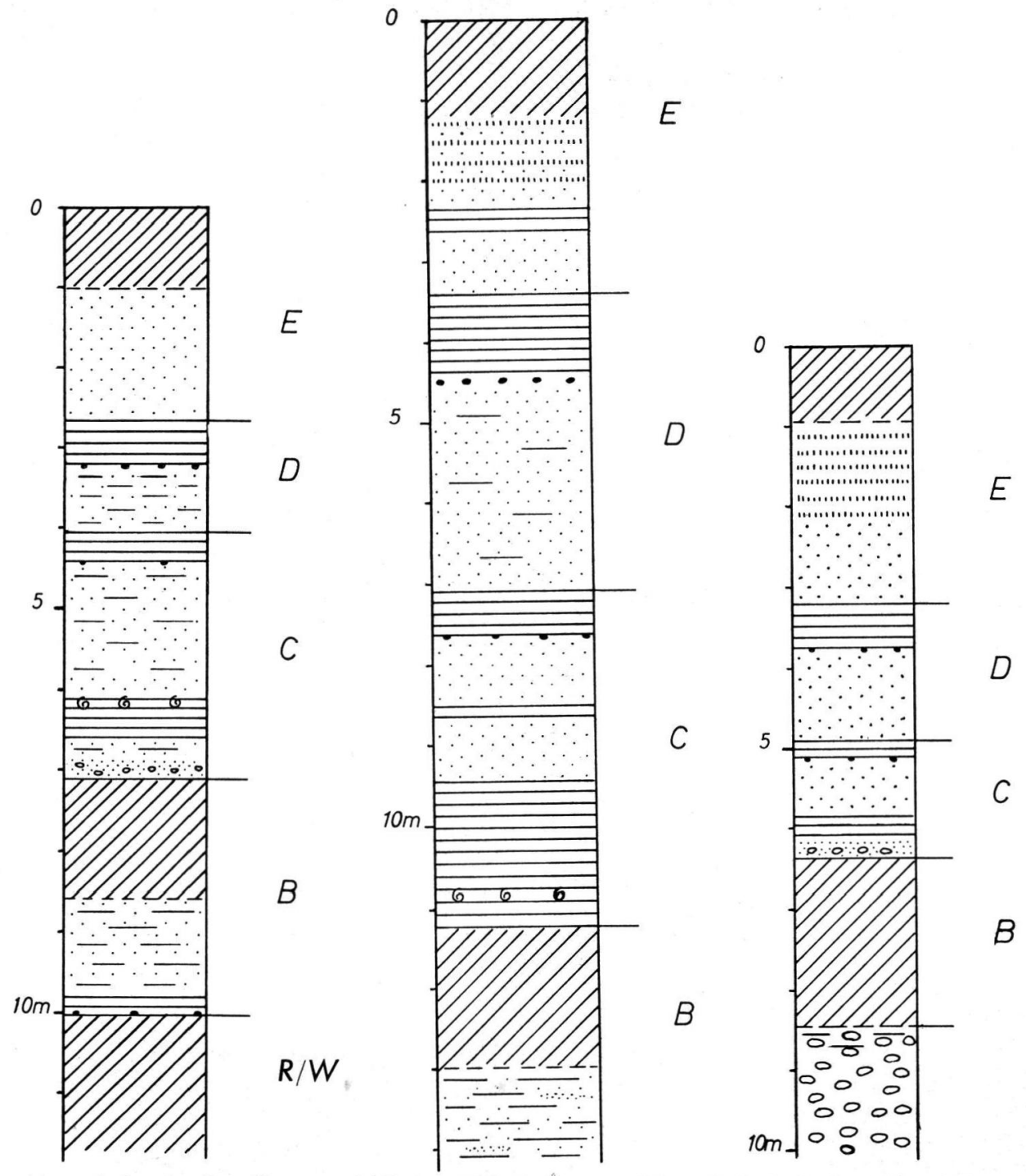

$B$

Abb. 5 (links): Lößprofil von Leichlingen, Zgl.-Grube. - Abb. 6 (Mitte): Lößprofil von Düsseldorf-Grafenberg, Zgl.-Gruben. - Abb. 7 (rechts): Lößprofil von Beggendorf, Zgl. Rosen.

8 Eiszeit und Gegenwart 


\section{Das Lößprofil in der Ziegeleigrube Leichlingen}

Ein recht vollständiges Profil im Würmlöß liegt am Westrande des Bergischen Landes in der Ziegeleigrube Leichlingen vor (Abb. 5):

E Bis $1,00 \mathrm{~m}$ Bodenbildung des Spätglazials und Holozäns - 2,65 m Löß (10 YR 6/4)

- 3,20 m schwach grauer kalkhaltiger Löß mit Eisenflecken, an der Basis Lößkindel: D Naßboden D 4,05 m streifiger, kalkhaltiger, feinsandiger Löß (10 YR 6/4)

- 4,40 m kräftig grauer Lößlehm mit vereinzelten Lößkindeln an der Basis, wenige Eisenflecken: Naßboden

C $\quad-6,15 \mathrm{~m}$ streifiger, $\mathrm{z}$. T. fleckiger, kalkhaltiger, feinsandiger Löß (10 YR 6/4)

- 6,60 m grauer, z. T. kräftig grauer Lößlehm mit Lößschnecken

- 7,10 m sandiger, kalkhaltiger Löß (10 YR 6/6), an der Basis Kieselsteinlage

- - - - - - - - - - - - - - - - - - - - - - -

- 8,60 m Parabraunerde, schwach pseudovergleyt

B-Horizont: feinsandiger Lehm mit Kieselsteinen (7,5 YR 5/6), Eisenmangankonkretionen.

B - 9,80 m kalkhaltiger feinsandiger Lößlehm mit sandigen Streifen (10 YR 6/4): Fließerde

- 10,05 m grauer kalkhaltiger Lößlehm mit Eisenflecken, an der Basis Lößkindel: Naßboden

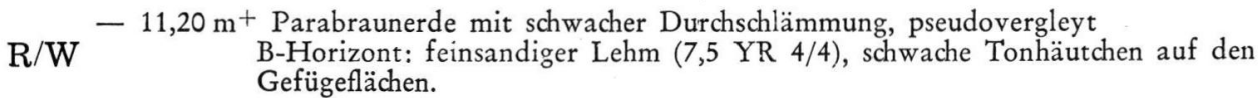

Das Profil dürfte auf Grund der Ergebnisse von Metternich im wesentlichen in die Würmzeit zu stellen sein. Zwischen die beiden Parabraunerden (R/W und $B$ ) ist hier ein Naßboden (9,80-10,05 m) eingeschaltet, der als Ergebnis eines neuerlichen kleinen Klimaumschwunges gedeutet wird. Die Naßbodenbildung mag durch das Bodenmaterial der Parabraunerde im Liegenden gefördert worden sein. Sie könnte so noch in die ausgehende Warmzeit gehören. Es ist jedoch wahrscheinlicher, daß in der ausgehenden Warmzeit das Bodenprofil gekappt worden ist und sich erst neuer Löß darüberlegte, der in einer späteren feuchten Phase zu einem Naßboden überprägt wurde. Für diese Auffassung spricht meines Erachtens die scharfe Abgrenzung des Naßbodens gegen die Parabraunerde und die Lößkindel an der Basis des Naßbodens. Die Pseudovergleyung der Parabraunerde könnte mit der Naßbodenbildung in Zusammenhang stehen, aber auch schon früher erfolgt sein. Ein neuerlicher kleiner Klimaumschwung ist daher für die Naßbodenbildung anzunehmen.

Im Mittelwürm herrschten Fließerden und Naßböden, und nur das Jungwürm weist noch primär windgelagerten Löß auf.

\section{Ziegeleigruben bei Diisseldorf-Grafenberg}

Der Löß liegt hier wie bei Leichlingen am Westhang des Bergischen Landes und wurde ehedem in drei Ziegeleigruben abgebaut, von denen heute nur in der mittleren der Abbau noch umgeht. Die jüngeren Bildungen sind am besten in der dem Hang zu gelegenen Grube zu erkennen, während in den Gruben zum Rhein hin die tieferen Schichten erschlossen sind. Das Profil ist aus den drei Gruben zusammengestellt (Abb. 6):

Bis $2,00 \mathrm{~m}$ Parabraunerde des Spätglazials und Holozäns; Flugsandeinlagerungen im unteren Teil

$\mathrm{E} \quad-2,40 \mathrm{~m} \mathrm{Löß} \mathrm{(10} \mathrm{YR} \mathrm{6/4)}$

- 2,65 $\mathrm{m}$ hellgrauer, feinsandiger Löß mit zahlreichen Eisenflecken: 'Naßboden

- $3,40 \mathrm{~m}$ Löß (10 YR 6/4) 
- 4,35 m kalkhaltiger, feinsandiger Lößlehm (10 YR 5/6), ? schwach humos, zahlreiche D Eisenflecken, an der Basis Lößkindel: Naßboden.

- 7,10 m Löß (10 YR 6/4), leicht streifig

— 7,65 m hellgrauer, feinsandiger Löß mit zahlreichen Eisenflecken: Naßboden

- 9,45 m Löß (10 YR 6/4)

C bei $8,6 \mathrm{~m}$ schwach graue Lößzone mit wenigen Eisenflecken

- 11,20 m kalkhaltiger, teils licht gelblich-brauner, teils grauer Löß mit zahlreichen Eisenflecken, an der Basis plattig und mit vielen Schneckenschalen: oben Naßboden, an der Basis Fließerde.

- 13,00 m Parabraunerde, pseudovergleyt 0,2 m A-Horizont: humoser, feinsandiger Lößlehm (10 YR 4/3) mit Eisenmangankonkretionen.

1,6 m B-Horizont: feinsandiger Lößlehm mit Kieselsteinen (7,5 YR 5/6).

$-14,20 \mathrm{~m}+$ feinsandiger Lößlehm mit Sandlinsen, zum Teil schichtig.

Bei diesem Profil sei auf den Naßboden (3,40-4,35 m) im Abschnitt D hingewiesen. Schwach verlehmtes Bodenmaterial (wahrscheinlich einer kalkhaltigen Braunerde) ist durch stärkere Nässeeinwirkung überprägt worden, so daß diese Bildung als Naßboden angesprochen wird. Durch seine Verbraunung und teilweise Beimengung von humosem Material ist dieser Horizont in sehr vielen Profilen wiederzuerkennen, so daß er recht gut durchverfolgt werden kann. In einigen Profilen ist er noch als kalkhaltige Braunerde entwickelt, meistens jedoch durch spätere Vernässung so stark überprägt, daß er als Naßboden bezeichnet wird.

Unter der Verwitterungsdecke des Spätglazials und Holozäns liegen zahlreiche deutlich braune Bänder, die die gleiche Färbung zeigen wie die Bodenbildung darüber. Korngrößenunterschiede zwischen den braunen Bändern und dem hellen Material sind schon im Gelände deutlich wahrzunehmen. Es handelt sich um Flugsandeinlagerungen. Ihre Verfärbung dürfte im Zusammenhang mit der jüngsten Bodenbildung stehen. Sie treten in sehr vielen Profilen des Niederrheingebietes auf.

\section{Ziegeleigrube Beggendorf SE Geilenkirchen}

Die Ziegeleigrube Rosen, Beggendorf, die bereits ganz im Westen des Untersuchungsgebietes liegt, zeigt die gleiche Gliederung, wie wir sie im übrigen Gebiet kennengelernt haben (Abb. 7):

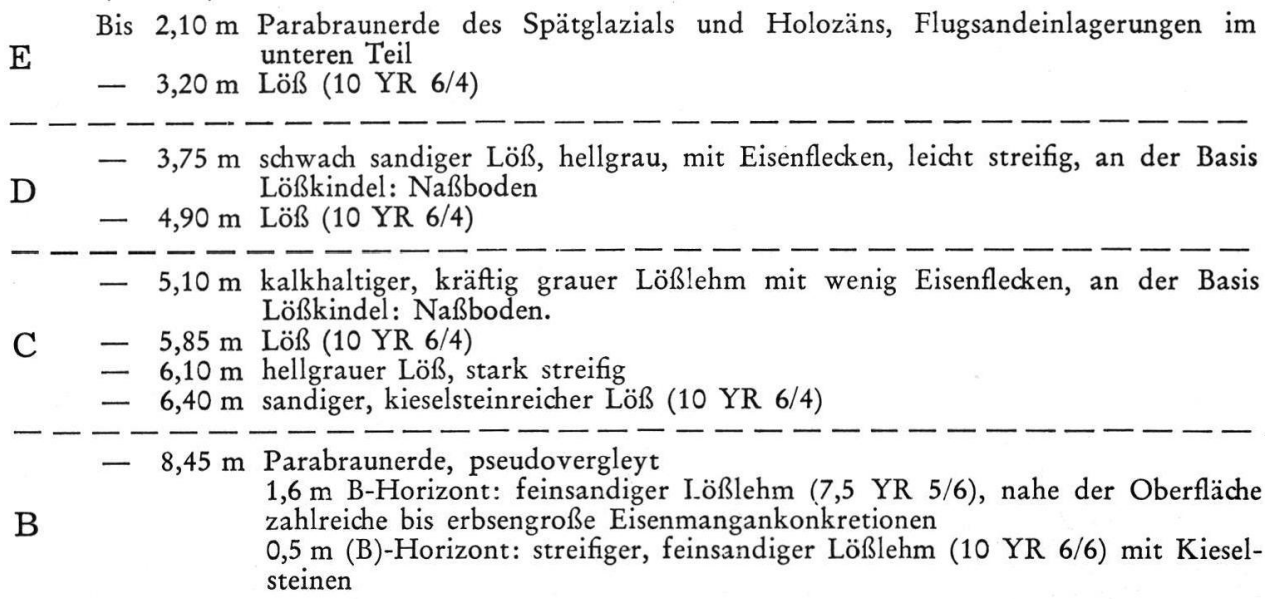

Bis 2,10 m Parabraunerde des Spätglazials und Holozäns, Flugsandeinlagerungen im unteren Teil

- $3,20 \mathrm{~m} \mathrm{Löß} \mathrm{(10} \mathrm{YR} \mathrm{6/4)}$

- 3,75 m schwach sandiger Löß, hellgrau, mit Eisenflecken, leicht streifig, an der Basis D Lößkindel: Naßboden

- 4,90 m Löß (10 YR 6/4)

$-5,10 \mathrm{~m}$ kalkhaltiger, kräftig grauer Lößlehm mit wenig Eisenflecken, an der Basis Lößkindel: Naßboden.

C $\quad-\quad 5,85 \mathrm{~m} \mathrm{Löß} \mathrm{(10} \mathrm{YR} \mathrm{6/4)}$

- 6,10 m hellgrauer Löß, stark streifig

- 6,40 m sandiger, kieselsteinreicher Löß (10 YR 6/4)

$--1,-15 \mathrm{~m}$ Parabraunerde, pseudovergleyt

1,6 m B-Horizont: feinsandiger I_ößlehm (7,5 YR 5/6), nahe der Oberfläche

B zahlreiche bis erbsengroße Eisenmangankonkretionen 0,5 m (B)-Horizont: streifiger, feinsandiger Lößlehm (10 YR 6/6) mit Kieselsteinen

$-10,00 \mathrm{~m}+$ Schottermaterial einer Hauptterrasse. 
Dieses Profil zeigt nichts Neues, wurde aber mit aufgenommen, weil es zum Lößgebiet des belgisch-französischen Raumes überleitet. Die Bodenbildungen des Mittelwürms sind hier alle als Naßböden entwickelt. Unter der Verwitterung des Spätglazials und Holozäns sitzen auch hier die braunen Bänder, die zum Hangenden hin stärker und dichter werden. Offenbar hat eine Verlagerung von Tonsubstanz von der Bodenbildung her stattgefunden (mündl. Mitt. W. PAAs).

\section{Die Böden in den Lößprofilen}

Als älteste Bodenbildung im Löß tritt gelegentlich eine Art Braunlehm auf, den W. PaAs (Diss. Bonn) genauer untersucht hat. Bislang wurde dieser Bodentyp dem Mindel/Riß-Interglazial zugeschrieben (mündl. Mitt. Prof. E. Mückenhausen). W. PaAs glaubt ihn dem Boden auf der Unteren Mittelterrasse gleichsetzen zu dürfen und stellt ihn daher in das Letzte Interglazial. Darüber folgen im allgemeinen zwei Parabraunerden, von denen die ältere meist eine geringere Durchschlämmung aufweist. Sehr oft zeigen die Parabraunerden noch die typische Gliederung mit den Horizonten $\mathrm{A}_{1} \mathrm{~A}_{3}$ und $\mathrm{B}$, so daß für einen großen Teil eine autochthone Lagerung als sicher angenommen werden kann. In vielen Profilen sind sie durch spätere Nässeeinwirkung pseudovergleyt.

Einen anderen Bodentyp stellen braune Verwitterungshorizonte dar, die hauptsächlich im höheren Teil der Profile auftreten. Es handelt sich um noch kalkhaltiges verbrauntes Bodenmaterial, das bei Umlagerung auch schwach humos sein kann. E. Schönhals (1951) bezeichnet diese Böden als kalkhaltige Braunerde. Oft liegen an ihrer Basis Lößkindel, so daß diese Braunerden sich offenbar noch in situ befinden.

Die Naßböden hat zuerst H. Freising (1951) aus Süddeutschland beschrieben, die nach ihm Ausdruck für ein kaltes und feuchtes Klima sind. Im rheinischen Löß kommen sie ebenso vor und können völlig isoliert im Löß auftreten oder vorangegangene Bodenbildungen überprägen. So läßt sich beobachten, daß nach einer schwachen Verlehmung (Bodenbildung) eine stärkere Vernässung folgte, so daß ein Horizont entstand, den man im Sinne von H. Freising als Naßboden ansprechen muß.

Der Naßboden allein, bzw. meist mit anschließender Fließerdebildung, kann Ausdruck einer Klimaschwankung sein. Es kann ihm aber auch eine wärmere und trockenere Phase vorangegangen sein, so daß er nur Teil eines Bodenzyklus ist. Der Klimaumschwung erfolgte dann von einer Warmzeit über eine kühle und feuchte Phase zu der kalten und trockeneren des folgenden Stadials. Naßböden und Fließerden sind Ausdruck der kühlen und feuchten Phase. Meistens verwischt aber die Fließerdebildung die vollständige Abfolge. Eine Untergliederung in eine erste Phase mit Tundren-Naßboden und eine zweite Phase mit auf noch stärkere Nässeeinwirkung weisender Fließerde nahm bereits K. Brunnacker (1956) vor.

Die Naßböden werden auch vom Verf. als Ergebnis einer Klimaschwankung aufgefaßt, wie das H. Freising schon getan hat. Diese Auffassung stützen faunistische Untersuchungen. Im Lößprofil von Kärlich (H. REMY 1959) z. B. entstammt Fauna 1 einem Naßbodenhorizont. Sie weist in ihrer Zusammensetzung auf eine Zeit größerer Feuchtigkeit, wie sie keinesfalls für ein Stadial mit Lößbildung typisch ist.

\section{Stratigraphische Deutung der Ablagerungen}

Für die stratigraphische Einordnung von Lößprofilen im Rheinland ist vor allem die Beziehung zu den Terrassen des Rheines und seiner Nebenflüsse entscheidend. Als einwandfrei datiert kann die Untere Mittelterrasse des Rheines gelten, die im Riß I (Drenthe) aufgeschottert wurde (vgl. die zusammenfassende Darstellung bei P. Woldstedt 1958, S. 56). Mit ihr gleichaltrig ist die Untere Mittelterrasse (=Talwegterrasse) der Mosel (vgl. E. KREMER 1954). Hier liegen auch zwei der vollständigsten Lößprofile im Unter- 
suchungsgebiet: Profil von Moselweiß bei Koblenz und das Profil von Metternich bei Koblenz in der ehemaligen Ziegeleigrube der Gebrüder Weglau.

Bei den Ausführungen über das Profil von Metternich wurde schon darauf hingewiesen, daß die Schotter der Unteren Mittelterrasse der Mosel eine kräftige Verwitterung tragen, die bodentypologisch dem Braunlehm nahesteht und etwas anderes darstellt als die Parabraunerden im Lößprofil darüber. Diese Terrassen-Verwitterung gehört in die Zeit nach dem Vorstoß des Saaleeises (Riß I). Für eine Einstufung in das Riß I/II-Interstadial ist die Verwitterung vielleicht $\mathrm{zu}$ intensiv. Diese müßte sonst im Interstadial stärker gewesen sein als im Riß/Würm-Interglazial, in das dann eine der Parabraunerden einzustufen wäre. So ist es zunächst wahrscheinlicher, daß die Braunlehmverwitterung in das Letzte Interglazial und der gesamte Löß darüber in das Würm zu stellen ist.

Die bodenkundlichen Überlegungen sprechen zunächst also gegen eine Einstufung der Verwitterung auf der uMT in eine Warmzeit, der man allgemein nur den Charakter eines Interstadials zubilligt (vgl. jedoch G. v. D. BRELIE 1955). In den rheinischen Lößprofilen liegen aber über den Ablagerungen der Saalevereisung noch drei fossile Bodenbildungen, die in "warmen" Zeiten entstanden sein müssen. Dabei gleichen die beiden Parabraunerden bodentypologisch den rezenten Böden im Untersuchungsgebiet. Wenn die Bodenbildung unmittelbar nach der Saalevereisung auch intensiver gewesen ist als die folgenden, so kann man sie deswegen nicht unbedingt dem letzten Interglazial zuordnen. Dagegen spricht. auch die Beobachtung von E. Mückenhausen (1939), daß die Böden des Warthestadiums denen der Weichselvereisung näherstehen. Es würde bei dieser Deutung auch jede Spur von Löß des Warthestadiums auf der uMT fehlen, den wir erwarten müßten. So scheint es nicht gerechtfertigt, das letzte Interglazial (Eem) in den Lößprofilen so tief anzusetzen, wie man dazu auf Grund des bodenkundlichen Befundes zunächst geneigt sein könnte. Es wird daher angenommen, daß der Boden auf der uMT in der Riß I/II-Warmzeit entstanden ist. Als letztinterglazialer Boden (Eem) wird im Untersuchungsgebiet die ältere fossile Parabraunerde aufgefaßt, zumal im Rheinland erst aus Ablagerungen ü be $r$ diesem Horizont Funde von Moustier-Artefakten vorliegen. Die Lößablagerungen mit ihren Böden darüber sind danach würmeiszeitlich.

Auf Grund der Bodentypologie gelangen wir also zu der Vorstellung, daß es nach der Saalevereisung mehrere „warme“ Zeiten gegeben hat. G. v. D BRELIE (1955) sieht auf Grund pollenanalytischer Untersuchungen in dem Interstadial Riß I/II ein echtes Interglazial („Ohe-Interglazial“), was mit dem bodenkundlichen Befund gut übereinstimmen würde. Wenn wir dann die ältere fossile Parabraunerde in das Eem-Interglazial stellen, für das eine ausgedehnte Bewaldung ähnlich der holozänen nachgewiesen ist, so sollte man auch für die Zeit, in der die jüngere fossile Parabraunerde entstanden ist, mindestens eine teilweise Wiederbewaldung erwarten.

In den meisten Lößprofilen ist demzufolge nur Würmlöß aufgeschlossen. Über den Rißlöß wissen wir noch sehr wenig. Wo er in geringer Mächtigkeit auftritt, ist er intensiv verwittert. Der Würm-Lößkomplex wird durch mehrere Bodenbildungen gegliedert. Bedeutende Wärmeschwankungen liegen in den Abschnitten ( $A$ und $B$ ) vor. Innerhalb dieser Abschnitte waren noch weitere Klimaschwankungen vorhanden, die sich in manchen rheinischen Lößprofilen als Naßböden abzeichnen. Das Auftreten derartiger Schwankungen in den Lößprofilen dürfte damit aber durchaus nicht vollständig sein.

Im Profil von Koblenz-Metternich ist im Abschnitt A Bodenmaterial erhalten, das zeitlich mit einer kalkhaltigen Braunerde in den Profilen von Koblenz-Moselweiß und Kärlich gleichgestellt wird. Ich glaubte seinerzeit im Profil von Kärlich (REMY 1959) diese Bodenbildung dem Göttweiger Boden gleichsetzen zu dürfen. Es scheint sich aber um einen Boden zu handeln, der zeitlich noch vor dem Göttweiger Boden im Altwürm (A) liegt.

Die Parabraunerde in B ist die letzte kräftige Bodenbildung im Würmlöß. Ich nehme daher an, daß es sich dabei um ein Aquivalent des Verlehmungshorizontes der Gött- 
weiger Bodenbildung handelt. Für diese Auffassung scheint mir auch das Auftreten von Moustier-Funden zu sprechen, die im Rheinlande in den Abschnitten A und B liegen. Diese untere Abteilung des Würmlösses wird daher auch im Sinne von P. Woldstedt (1960) als Altwürm bezeichnet.

Dem als Altwürm benannten Zeitraum steht im oberen Teil der Profile ein Lößkomplex gegenüber, der von weniger mächtigen Böden gegliedert und als Mittel- $(C+D)$ und Jungwürm (E) aufgefaßt wird. Den basalen Teil (C) gliedern bis zu drei Naßböden, die aber durch stärkere Fließvorgänge unterdrückt werden können, so daß öfter nur ein \pm mächtiger Fließlöß auftritt. In vielen Profilen ist gröberer Schutt eingelagert, in manchen fluviatiler Sand.

In den Bodenbildungen des Abschnittes $C$ scheinen ähnliche Wärmeschwankungen vorzuliegen, wie sie in österreichischen Profilen die Schwarzerdehorizonte über der Göttweiger Verlehmungszone darstellen und im Komplex „Stillfried A“ (FINK 1954) zusammengefaßt werden. Der Unterschied liegt in der Bodenausbildung: im Rheinland sind es bis zu drei Naßböden, von denen in wenigen Aufschlüssen wohl der mittlere als kalkhaltige Braunerde entwickelt sein kann.

Ein oft sehr deutlich ausgeprägter Verlehmungshorizont (D) (kalkhaltige Braunerde im Sinne von E. SchöNhals) durchzieht den jüngeren Würmlöß. Er ist in Metternich Träger der Spätaurignac-Kultur und durch eine Molluskenfauna im Profil von Kärlich (H. REMY 1959) als verhältnismäßig warme Schwankung gekennzeichnet. Dieser Horizont scheint besonders im Neuwieder Becken und im nördlichen Niederrheingebiet ausgeprägt zu sein. Seine Mächtigkeit schwankt um 0,5 m.

Ganz ähnlich liegen die Verhältnisse in den Profilen von Unterwisternitz und Pollau, wo über einer schwachen Verlehmung, die als Paudorfer Horizont aufgefaßt wird, das Gravette auftritt. Es liegt daher nahe, das Gleiche auch für das Rheinland anzunehmen und den Verlehmungshorizont (D) mit der Paudorfer Verlehmung zu parallelisieren.

Mit der neuen Lößperiode läßt P. Woldstedt (1960) das Jungwürm (E) beginnen. Dieses unterteilt im Rheinland noch ein schwacher, oft unterdrückter Bodenhorizont. Er tritt teils als ganz schwache Verlehmung (Metternich, Garzen II) oder als Naßboden (Grafenberg) auf. An seiner Oberfläche liegt in Metternich eine Fauna (Pupilla-Horizont), die auch auftreten kann, ohne daß Anzeichen einer Bodenbildung vorhanden sind. Diese Faunen werden vorerst jedenfalls als gleichalt aufgefaßt. In diesem Komplex E liegt auch ein Tuffbändchen, das nach J. FRECHEN einem Ausbruch bei Kärlich entstammt und weithin nach $S E$ zu verfolgen ist.

Der jüngste Löß trägt die Böden des Spätglazials und Holozäns, die als Braunerden und Parabraunerden entwickelt sind. Gegen Ende der Lößsedimentation entstand durch Schwankungen in der Transportkraft des Windes eine Bänderung, die sich heute in sehr vielen Profilen durch die Verwitterung als braune Streifung an der Basis der jüngsten Bodenbildung abhebt.

\section{Kulturreste im rheinischen Löß}

Die Moustier-Funde von Rheindahlen im Niederrheingebiet (K. NARR 1951) gehören wahrscheinlich dem Altwürm an. Fine Moustier-Handspitze von dreieckiger Form ist in der Zgl. Friedhofen in Metternich bei Koblenz gefunden worden. Sie lag nach Angaben von A. GüNTher in oder auf einem Bodenhorizont, der mit der jüngsten fossilen Parabraunerde der Zgl. Weglau in Metternich (B) gleichgestellt werden kann. Damit würde dieser Fund etwa in die Warmzeit zwischen Alt- und Mittelwürm gehören und etwas jünger sein als die Moustierfunde von Rheindahlen.

Erheblich jünger sind die Funde in der Grube Weglau bei Metternich. Sie gehören dem Spätaurignac (Gravette) an und liegen auf dem Verlehmungshorizont des Abschnittes D (Ende von Mittelwürm). Diese Funde lassen sich dank der ausgezeichneten Aufnahme von A. Günther (1907) und H. Hofer (1937) stratigraphisch sicher einstufen. 
Recht unsicher sind die Funde von Rhens. Ihre Lage im Profil konnte A. Günther damals nicht mehr ermitteln. Es soll sich um Werkzeuge des gleichen Typs handeln wie bei Metternich. Die Fundschicht scheint aber tiefer gelegen zu haben als in Metternich. Ebenso unsicher bleibt die stratigraphische Zuordnung der Funde von Kärlich, die durch einen Ausbläser in jüngere Schichten getragen worden sind.

An das Ende der Lößperiode am Mittelrhein gehören die Funde der Magdalen-Kultur vom Martinsberg bei Andernach.

\section{Vulkanische Einlagerungen}

In den Lößablagerungen des Mittelrheingebietes sind vulkanische Einlagerungen nicht selten. Sie haben ihren Ursprung in der Eifel, von wo sie über weite Gebiete getragen worden sind. Die größte Verbreitung hat ein Aschenbändchen im höheren Teil der Profile, das einem Ausbruch bei Kärlich entstammt. Dieses Aschenbändchen tritt in einem weiten, SE gerichteten Streukegel bis nach Süddeutschland auf und liegt oft über einer schwachen Verlehmungszone (D) im jüngsten Löß.

Im Profil von Kärlich ist auch in den tieferen Lößschichten bereits vulkanisches Material eingelagert (E. A. Rosauer 1957, J. Frecien \& E. A. Rosauer 1959), das aber bisher nicht in anderen Lößprofilen nachgewiesen werden konnte.

Der Ausbruch der jüngeren Aschen des Rodderberges bei Bonn erfolgte gegen Ende des Mittelwürms (H. REmy 1959). Er liegt etwas vor der Wärmeschwankung, die durch die Spätaurignac-Kultur von Metternich charakterisiert wird (D). Die Verbreitung der Aschen läßt sich nur wenige Kilometer nach $\mathrm{N}$ nachweisen.

In vielen Lößprofilen am Mittelrhein liegt auf dem jüngsten Löß Bimstuff, der dem Ausbruch des Laacher-See-Kessels entstammt.

Tabelle 1

Die zeitliche Aufeinanderfolge von Böden, Kulturen und vulkanischen Ereignissen im Würm'öß am Mittel- und Niederrhein.

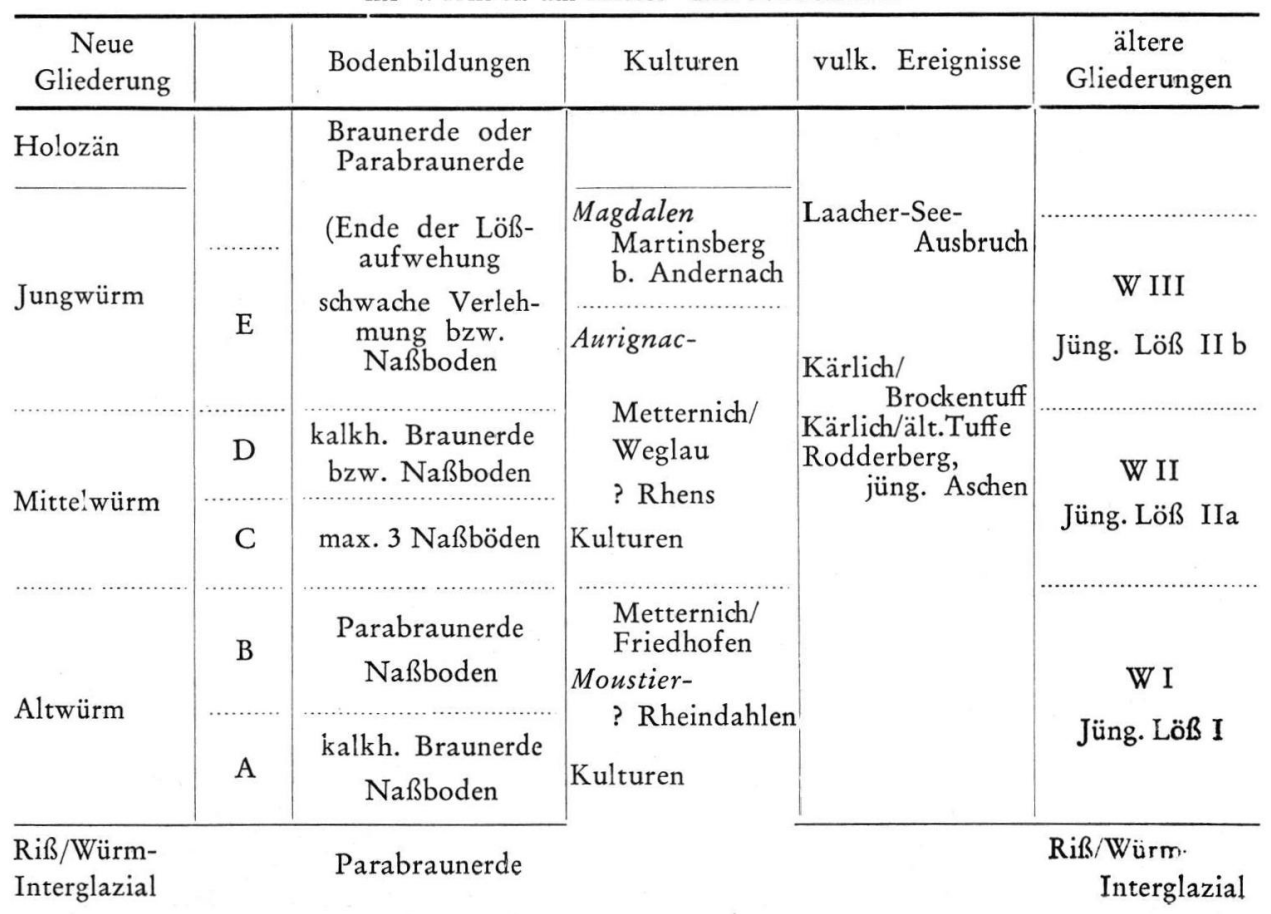




\section{Lit eratur}

v. D. Brelie, G.: Die pollenstratigraphische Gliederung des Pleistozäns in Nordwestdeutschland. Eiszeitalter u. Gegenwart 6, S. 25-38, Ơhringen 1955.

BRUnNACKer, K.: Würmeiszeitlicher Löß und fossile Böden in Mainfranken. - Geologica Bavarica 25, S. 27-43, München 1956.

FinK, J.: Die fossilen Böden im österreichischen Löß. - Quartär 6, S. 85-107, 1954.

Frechen, J. \& Rosauer, E. A.: Aufbau und Gliederung des Würm-Löß-Profiles von Kärlich im Neuwieder Becken. - Fortschr. Geol. Rheinld. u. Westf. 4, Krefeld 1959.

Freising, H.: Neue Ergebnisse der Lößforschung im nördlichen Württemberg. - Jh. geol. Abt. württemb. statist. L. A. 1, S. 54-59, Stuttgart 1951.

GüNTHER, A.: Paläolithische Fundstellen im Löß bei Koblenz. - Bonner Jahrbücher 116, S. 344 bis 362, Bonn 1907.

Hofer, H.: Neue Beobachtungen an mittelrheinischen Iößstationen. - Nachr.-Bl. dtsch. Vorzeit 13, S. 101-103, Leipzig 1937.

Kremer, E.: Die Terrassenlandschaft der mittleren Mosel als Beitrag zur Quartärgeschichte. Arbeiten zur Rheinischen Landeskunde, H. 6, Bonn 1954.

MÜckenhausen, E.: Fossile Böden im nördlichen Rheinland. - Zeitschr. f. Pflanzenernährung, Düngung, Bodenkunde 65, S. 81-103, Weinheim/Bergstr. u. Berlin 1954. - - Die stratigraphische Gliederung des Lößkomplexes von Kärlich im Neuwieder Becken. - Fortschr. Geol. Rheinld. u. Westf. 4, S. 283-300, Krefeld 1959.

Mǘckenhausen, E.: Die Böden des Warthestadiums in Nordhannover im Vergleich zum westlichen Alt- und zum östlichen Jungdiluvium. - Abh. nat. Ver. Bremen 31, S. 334-346, Bremen 1939.

NARR, K.: Alt- und mittelpaläolithische Funde aus rheinischen Freilandstationen. - Bonner Jahrbücher 151, S. 5-51, Bonn 1951.

Remy, H. \& PaAs, W.: Die Lößprofile von Koblenz-Metternich und Moselweiß. - Fortschr. Geol. Rheinld. u. Westf. 4, S. 331-336, Krefeld 1959.

REmY, H.: Zur Gliederung des Lösses am unteren Mittelrhein mit besonderer Berücksichtigung der Faunen. - Fortschr. Geol. Rheinld. u. Westf. 4, S. 323-330, Krefeld 1959. - - Die zeitliche Stellung der Rodderbergtuffe im rheinischen Löß. - Decheniana 112, S. 271-278, Bonn 1960.

Rosauer, E. A.: Über die Klimabedingungen der eiszeitlichen Lößbildung (engl.). - Diss. Bonn 1957.

SchöNHALS, E.: Uber fossile Böden im nichtvereisten Gebiet. - Eiszeitalter u. Gegenwart 1, S. 109 bis 130, OOhringen 1951.

Woldstedt, P.: Das Eiszeitalter, 2. Aufl. Bd. II. - Stuttgart 1958. - - Die Letzte Eiszeit in Nordamerika und Europa. - Eiszeitalter u. Gegenwart 11, 1960.

Manuskr. eingeg. 22. 8. 1960.

Anschrift des Verf.: Dr. Horst Remy, Geologisches Institut der Universität, Bonn, Nußallee 2. 